Abbildung 2.

Überleben von Patienten mit NSCLC im Stadium I nach Operation und ohne adjuvante Therapie in Abhängigkeit von der Expression der Thymidylat-Synthase. Nach Zheng Z et al. Cancer 2008;112:2770.

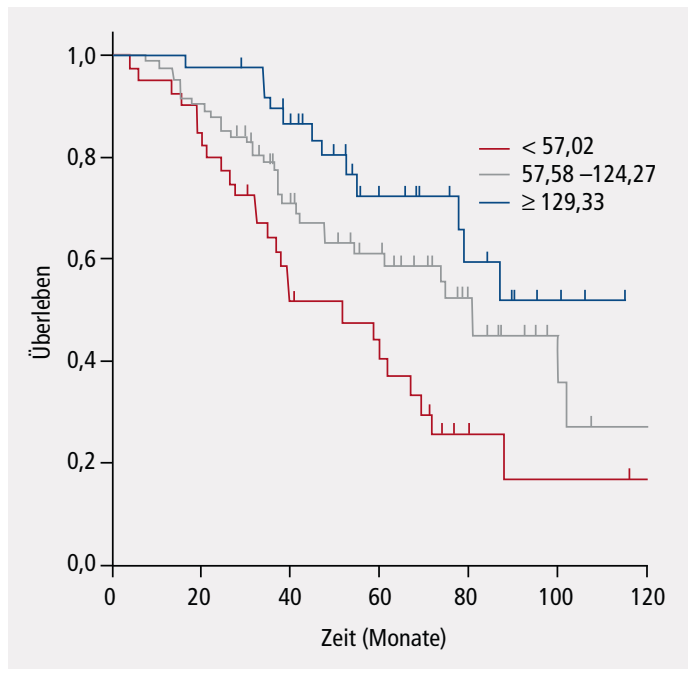

beider Enzyme im Resektat niedrig ist, hingegen keine Therapie bei hoher Expression. Und in einer britischen Phase-III-Studie erhalten Patienten mit fortgeschrittenen Nicht-Plattenepithel-Tumoren in Abhängigkeit vom ERCC1-Expressionsstatus Pemetrexed entweder mit Cisplatin oder mit Paclitaxel. Weitere Studien in Japan, Griechenland und Frankreich zu dieser Fragestellung sind ebenfalls angelaufen.

\section{Thymidylat-Synthase prädiktiv?}

Pemetrexed ist nur bei NSCLC mit Nicht-Plattenepithel-Histologie zugelassen, aber auch hier stellt sich die Frage, ob eine molekulare Variabilität der Tumoren für die Wirkung des Medikaments von Bedeutung ist. Pemetrexed greift in den PurinStoffwechsel ein und hemmt neben zwei weiteren Enzymen die Thymidylat-Synthase (TS). Die Expression der TS korreliert bei Patienten mit Tumoren des Stadiums I, die keine adjuvante Therapie erhalten, in retrospektiven Auswertungen signifikant mit dem Überleben (Abb. 2). „Die Frage, ob die TS-Expression, die zum Beispiel starke Unterschiede zwischen Adeno- und Plattenepithelkarzinomen zeigt, für die klinische Wirkung von Pemetrexed bedeutsam ist und damit hier möglicherweise einen prädiktiven Marker darstellt, ist noch ungeklärt", so Bepler. Ebenso unklar sei, ob zur Bestimmung der Expression immunhistochemische Methoden oder besser molekulargenetische Verfahren auf Basis der Polymerasekettenreaktion eingesetzt werden sollten, fügte Bepler hinzu.

\section{Josef Gulden}

Quelle: 29. Deutscher Krebskongress vom 24.-27. Februar 2010 in Berlin
Fleur-Hiege-Habilitationsstipendium der Hiege-Stiftung gegen Hautkrebs

2010 vergibt die Hiege-Stiftung gegen Hautkrebs ein 12-monatiges Habilitationsstipendium. Es richtet sich an junge Nachwuchswissenschaftler/innen, die noch am Anfang ihrer wissenschaftlichen Karriere stehen, aber bereits über Erfahrungen in experimenteller oder klinischer Forschung verfügen. Ihr Tätigkeitsschwerpunkt sollte im Bereich der Erforschung von Hauttumoren, vorzugsweise des malignen Melanoms, liegen. Das Habilitationsstipendium ist im Sinne einer Anschubfinanzierung für die Freistellung von klinischen Routinetätigkeiten zur Erstellung einer Habilitationsschrift gedacht. Die Bewerberin/der Bewerber sollte nachgewiesenermaßen die akademische Reife zum Anfertigen einer Habilitationsschrift besitzen und dies von dem/der entsprechenden Klinikdirektor/in bescheinigt bekommen.

Eine Ko-Finanzierung bereits geförderter Projekte wird nicht unterstützt. Das 12-monatige Stipendium wird mit 25.000 Euro für eine Forschungsbefreiung im Inland gewährt.
Es wird vorausgesetzt, dass das Klinikum den Bewerber/die Bewerberin zusätzlich finanziell unterstützt.

Folgende Bedingungen sollten erfüllt sein:

- Bewerben können sich sehr gut ausgewiesene, promovierte Mediziner/innen, welche eine akademische Laufbahn planen, jedoch noch nicht habilitiert sind.

- Das Alter der Bewerber/innen liegt im Regelfall unter 40 Jahren. Das Stipendium wird für ein Jahr gewährt.

- Bewerbungen sind bis zum 30.06.2010 bzw. bis zum 30.06. der Folgejahre einzureichen. Der nächste Bezugstermin des Fleur-Hiege-Habilitationsstipendiums ist der 01.10.2010.

\section{Forschungsprojekte der Hiege-Stiftung gegen Hautkrebs}

Die Hiege-Stiftung gegen Hautkrebs vergibt jährlich mehrere Forschungsprojekte im Sinne einer Anschubfinanzierung in der Größenordnung von $10.000-20.000$ Euro. Anträge können von Wissenschaftlern gestellt werden, die in der Hautkrebsforschung ausgewiesen sind und ein neues Projekt beginnen möchten, das anderweitig noch nicht finanziert ist. Die Infrastruktur zur Umsetzung des beantragten Projektes muss von der Institution des Antragsstellers schriftlich bestätigt werden. Bewerberinnen und Bewerber können sich bis zum 30.06. eines jeden Jahres bei der Hiege-Stiftung gegen Hautkrebs, Parkallee 43, 20144 Hamburg mit folgenden Unterlagen bewerben:

- Curriculum Vitae mit Publikationsliste.

- Darstellung des Forschungsvorhabens.

- Die Forschungsarbeiten sollten ganz oder weitgehend an einer deutschen Klinik oder einem deutschen Institut angefertigt werden.

- Acht Ausdrucke der vorgenannten Unterlagen sollten beigelegt werden.

Weitere Informationen zu den Zielen der Hiege-Stiftung gegen Hautkrebs im Internet: www.hiege-stiftung-gegen-hautkrebs.de 